\title{
Metabolic engineering of Saccharomyces cerevisiae for efficient production of glucaric acid at high titer
}

\author{
Na Chen ${ }^{1,2}$, Jingya Wang ${ }^{1,2}$, Yunying Zhao ${ }^{1,2^{*}}$ and Yu Deng ${ }^{1,2^{*}}$ (D)
}

\begin{abstract}
Background: Glucaric acid is a high-value-added chemical that can be used in various fields. Because chemical oxidation of glucose to produce glucaric acid is not environmentally friendly, microbial production has attracted increasing interest recently. Biological pathways to synthesize glucaric acid from glucose in both Escherichia coli and Saccharomyces cerevisiae by co-expression of genes encoding myo-inositol-1-phosphate synthase (Ino1), myo-inositol oxygenase (MIOX), and uronate dehydrogenase (Udh) have been constructed. However, low activity and instability of MIOX from Mus musculus was proved to be the bottleneck in this pathway.

Results: A more stable miox4 from Arabidopsis thaliana was chosen in the present study. In addition, high copy deltasequence integration of miox 4 into the $S$. cerevisiae genome was performed to increase its expression level further. Enzymatic assay and quantitative real-time PCR analysis revealed that delta-sequence-based integrative expression increased MIOX4 activity and stability, thus increasing glucaric acid titer about eight times over that of episomal expression. By fed-batch fermentation supplemented with $60 \mathrm{mM}(10.8 \mathrm{~g} / \mathrm{L})$ inositol, the multi-copy integrative expression S. cerevisiae strain produced $6 \mathrm{~g} / \mathrm{L}(28.6 \mathrm{mM})$ glucaric acid from myo-inositol, the highest titer that had been ever reported in S. cerevisiae.

Conclusions: In this study, glucaric acid titer was increased to $6 \mathrm{~g} / \mathrm{L}$ in S. cerevisiae by integrating the miox 4 gene from $A$. thaliana and the udh gene from Pseudomonas syringae into the delta sequence of genomes. Delta-sequencebased integrative expression increased both the number of target gene copies and their stabilities. This approach could be used for a wide range of metabolic pathway engineering applications with $\mathrm{S}$. cerevisiae.
\end{abstract}

Keywords: Glucaric acid, Metabolic engineering, Saccharomyces cerevisiae, miox4, Delta-sequence integration

\section{Background}

Glucaric acid, which was one of the "top value-added chemicals from biomass" announced by the US Department of Energy in 2004 [1], has been studied for therapeutic uses in cholesterol reduction [2] and cancer therapy [3] as well as for use as a building block for polymers like nylon [4]. Currently, glucaric acid is produced mainly by chemical oxidation of glucose, with nitric acid as solvent and oxidant [5], which has led to low yields and

\footnotetext{
*Correspondence: yunyingzhao@jiangnan.edu.cn;

dengyu@jiangnan.edu.cn

${ }^{1}$ National Engineering Laboratory for Cereal Fermentation Technology (NELCF), Jiangnan University, 1800 Lihu Road, Wuxi 214122, Jiangsu, China Full list of author information is available at the end of the article
}

toxic byproducts. Therefore researchers have been showing more interest in microbial production of glucaric acid in an effective and environmentally friendly manner.

Moon et al. [1] constructed a biological pathway to produce glucaric acid from glucose in Escherichia coli by coexpression of genes encoding myo-inositol-1-phosphate synthase (Ino1) from Saccharomyces cerevisiae, myoinositol oxygenase (MIOX) from Mus musculus, and uronate dehydrogenase (Udh) from Pseudomonas syringae. Analysis of this metabolic pathway revealed that MIOX was rate-limiting, leading to a low glucaric acid titer of $0.72 \mathrm{~g} / \mathrm{L}$. To increase D-glucaric acid titer in E. coli, novel synthetic tools like synthetic scaffolds and a N-terminal SUMO fusion were used to increase MIOX activity. 
These two engineered strains produced 2.5 and $4.75 \mathrm{~g} / \mathrm{L}$ $\mathrm{D}$-glucaric acid from $10 \mathrm{~g} / \mathrm{L}$ glucose and $60 \mathrm{mM}$ myoinositol $[6,7]$. However, it appeared that a D-glucaric acid titer above $5 \mathrm{~g} / \mathrm{L}$ would inhibit further production in $E$. coli due to a pH-mediated effect. Gupta et al. [8] transferred the synthetic glucaric acid pathway from $E$. coli to S. cerevisiae and found that myo-inositol availability for the At strain (S. cerevisiae containing miox4 gene from Arabidopsis thaliana) and MIOX activity for the Mm strain (S. cerevisiae containing MIOX from M. musculus) were rate-limiting. Maximum titer in the At strain was $1.6 \mathrm{~g} / \mathrm{L}$ glucaric acid from glucose supplemented with myo-inositol. Liu et al. [9] constructed a glucaric acid synthetic pathway in Pichia pastoris using MIOX from P. pastoris itself or mice and Udh from Pseudomonas putida, resulting in glucaric acid titers of $107.19 \pm 11.91$ and $785.4 \pm 1.41 \mathrm{mg} / \mathrm{L}$ respectively.

Considering the relatively low glucaric acid titers in E. coli and S. cerevisiae, this study aimed to increase glucaric acid titer in S. cerevisiae further by integrating miox4 from $A$. thaliana $[10,11]$ into a multi-copy delta sequence of genomes. There are at least three reasons for the choice of baker's yeast: (1) S. cerevisiae has better acid tolerance than E. coli; (2) as an eukaryotic organism, $S$. cerevisiae has a post-transcription system that may be advantageous for more stable expression of the miox4 gene derived from $A$. thaliana; and (3) some yeast strains have been widely used in the study of organic compound production both for their safety and their sophisticated genetic engineering toolkit [12]. This toolkit includes the organic acid, malic acid, and succinic acid produced by engineered $S$. cerevisiae $[13,14]$ and the fatty acidbased fuels and lipids synthesized by Y. lipolytica $[15,16]$. Delta-sequence-based constitutive expression increased both the number of target gene copies and their stabilities [17]. This approach could be used for a wide range of metabolic pathway engineering applications of $S$. cerevisiae (Fig. 1).

\section{Methods}

\section{Strains and culture conditions}

All strains and plasmids are shown in Table 1, and the primers used in this study are listed in Additional file 1: Table S1. E. coli JM109 cells were cultured in LB medium $[1 \%(\mathrm{w} / \mathrm{v})$ tryptone, $0.5 \%(\mathrm{w} / \mathrm{v})$ yeast extract, and $1 \%(\mathrm{w} / \mathrm{v})$ $\mathrm{NaCl}]$ at $37^{\circ} \mathrm{C}$. Yeast cells were cultured in YPD medium $[2 \%(\mathrm{w} / \mathrm{v})$ tryptone, $1 \%(\mathrm{w} / \mathrm{v})$ yeast extract, and $2 \%(\mathrm{w} / \mathrm{v})$ sodium chloride] or SD-URA/SD-HIS medium [0.17\% $(\mathrm{w} / \mathrm{v})$ yeast nitrogen base, $2 \%(\mathrm{w} / \mathrm{v})$ glucose, $0.5 \%$ ammonia sulfate, adding $1 / 10 \mathrm{~mL}$ amino acid mixture without uracil or histidine] at $30{ }^{\circ} \mathrm{C}$. Solid media were produced by adding $2 \%(\mathrm{w} / \mathrm{v})$ agar if necessary.
For shake flask cultivation, $50 \mathrm{~mL}$ of YPD medium with or without $10 \mathrm{~g} / \mathrm{L}$ myo-inositol in a $250 \mathrm{~mL}$ shake flask was used as the initial fermentation medium. Cultures were first grown overnight at $30{ }^{\circ} \mathrm{C}$ and $250 \mathrm{rpm}$ in YPD medium with myo-inositol and then inoculated to a density at $600 \mathrm{~nm}\left(\mathrm{OD}_{600}\right)$ of 0.05 . Cultures were incubated at $30^{\circ} \mathrm{C}$ and $250 \mathrm{rpm}$ for about 7 days.

\section{Plasmid construction}

The miox4 [10] gene encoding MIOX4 enzyme in $A$. thaliana and the $u d h$ gene encoding Udh in P. syringae [18] were codon-optimized and synthesized by Genewiz (Suzhou, China). To construct the pY26-miox4 plasmid, the miox4 gene was first digested with BglII/NotI and then ligated to the pY26-GPD-TEF plasmid [19], which was digested with $B g l \mathrm{II} /$ Not I to generate the pY26-miox4 plasmid. The $u d h$ gene was ligated to pY26-miox4 using the Gibson Assembly method to construct the pY26miox4-udh plasmid. The miox4 gene with $6 \times$ his-tag was PCR-amplified, digested with EcoRI/XhoI, and ligated to EcoRI/XhoI-digested pY26-GPD-TEF to generate the pY26-miox4-6 $\times$ His plasmid expressing the MIOX4$6 \times$ His fusion protein.

\section{Genetic manipulations}

To knock out the OPI1 $[20,21]$ gene, which was functional in the negative regulation of Inositol-1-phosphate synthase (Ino1), the loxP-kan-loxP cassette [22] containing the upstream and downstream $50 \mathrm{bp}$ of the OPI1 gene was amplified by PUG $6 \mathrm{~F}$ and PUG $6 \mathrm{R}$ primers from the pUG6 plasmid and transformed into S. cerevisiae BY4741-competent cells through the Li-Ac method [23]. The kanMX selection cassette was cured from successful deletion mutants using Cre recombinase expressed by the pSH47 [24] plasmid. Then the deletion mutant was subcultured continuously for loss of pSH47, generating the BY4741 opi1 1 strain. To integrate exogenous genes into the genome, delta1 and delta2 $[25,26]$ fragments were amplified from the S. cerevisiae genome using genF-2/ genR-2 and genF-5/genR-5 primers respectively, and the miox 4 and $u d h$ expression cassettes TMA and GUC were amplified from the pY26-miox4-udh plasmid by genF-3/ genR-3 and genF-4/genR-4 primers respectively. In addition, two parts of the selection marker HIS3, HIS3-1 and HIS3-2, were amplified from pRS313 using genF-1/ genR-1 and genF-6/genR-6. The above PCR products were gel-extracted, and touchdown PCR was used to assemble two homologous arms containing delta1, TMA, HIS3-1 and HIS3-2, GUC, and delta2. Then the two homologous arms were transformed into a solid medium lacking histidine, and the high glucaric acid-producing strain was screened. 


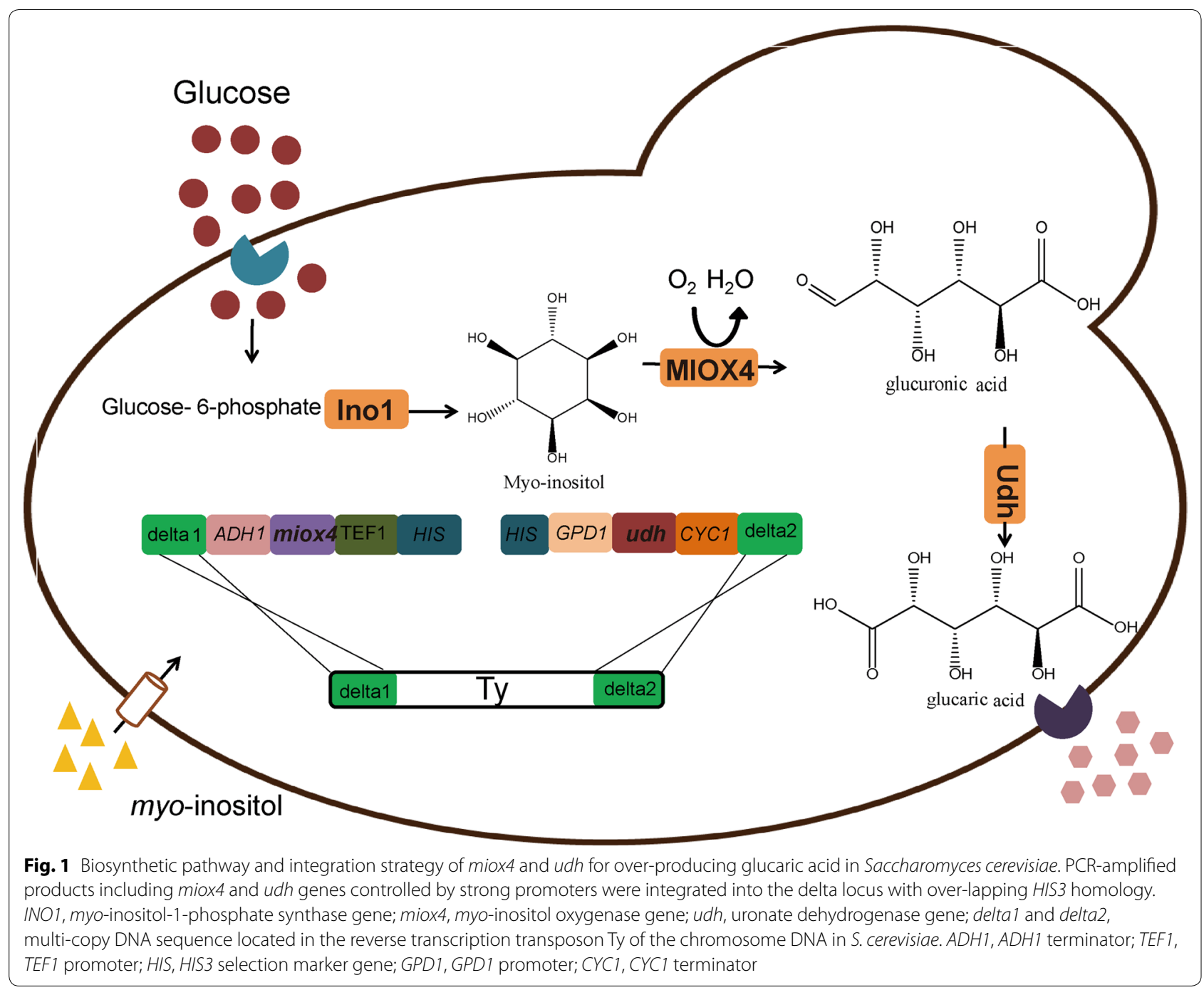

Table 1 Strains and plasmids used in this study

\begin{tabular}{|c|c|c|}
\hline Strains and plasmids & Relevant genotype & Reference \\
\hline E. coli JM109 & & Stored in author's laboratory \\
\hline S. cerevisiae BY4741 & $M A T a$, his $3 \Delta 1$, leu2 $\triangle 0$, met15 $\Delta 0$, ura3 $\Delta 0$ & Stored in author's laboratory \\
\hline $\begin{array}{l}\text { BY4741 opi1 } \\
\text { Bga-0 }\end{array}$ & $\begin{array}{l}\text { MATa, his } 3 \Delta 1 \text {, leu2 } \triangle 0 \text {, met } 15 \Delta 0 \text {, ura3 } \triangle 0 \text {, opi1 } \triangle 0 \\
\text { BY474 carrying pY26GPD-TEF-miox4-udh }\end{array}$ & $\begin{array}{l}\text { Stored in author's laboratory } \\
\text { This study }\end{array}$ \\
\hline Bga-1 & BY474 opi1 $\triangle$ carrying pY26GPD-TEF-miox4-udh & This study \\
\hline Bga-2 & BY474 opi1 $\triangle$ carrying pY26GPD-TEF-miox4-histag & This study \\
\hline Bga-3 & BY474 opi $1 \Delta$ carrying miox4 and $u d h$ & This study \\
\hline E. colipUG6 & E. coli plasmid with segment LoxP-KanMX4-LoxP & Stored in author's laboratory \\
\hline $\begin{array}{l}\text { E. coli pSH65 } \\
\text { pY26-GPD-TEF } \\
\text { pY26-miox4-udh } \\
\text { pY26-miox4-histag }\end{array}$ & $\begin{array}{l}\text { Shuttle plasmid for E. coli and S. cerevisiae, Cre gene } \\
\text { under GAL2 regulative regulation } \\
\text { Integrating plasmid, URA3 marker, MCS derived from } \\
\text { pBLUESCRIPT (ColE1 (derivative) ori, f1 ori, Amp }{ }^{R} \text { ) } \\
\text { pY26GPD-TEF carrying miox4 and udh } \\
\text { pY26GPD-TEF carrying miox4-histag }\end{array}$ & $\begin{array}{l}\text { Stored in author's laboratory } \\
\text { Stored in author's laboratory } \\
\text { This study } \\
\text { This study }\end{array}$ \\
\hline
\end{tabular}




\section{MIOX4 activity assay and immunoblot analysis}

Bga-2 containing pY26-miox4- $6 \times$ His plasmid was cultured in SD-Ura medium at $30{ }^{\circ} \mathrm{C}$ in a rotary shaker at $250 \mathrm{rpm}$ up to $\mathrm{OD}_{600}$ of $0.6-0.8$, then inoculated into $50 \mathrm{~mL}$ of SD-Ura medium using S. cerevisiae BY4741 containing pY26-GPD-TEF plasmid as a control. After $24 \mathrm{~h}$ cultivation, cell pellets were harvested and re-suspended in $50 \mathrm{mM}$ Tris- $\mathrm{HCl}$ buffer ( $\mathrm{pH} 7.0)$, then disrupted by ultrasonication and centrifuged at $13,000 \times g$ for $10 \mathrm{~min}$. The supernatant was used for the enzymatic activity assay. The reaction system $(200 \mu \mathrm{L})$ contained $50 \mathrm{mM}$ Tris- $\mathrm{HCl}$ buffer ( $\mathrm{pH} 7.0$ ), $2.0 \mathrm{mM}$ L-cysteine, $1.0 \mathrm{mM}$ ammonium ferrous sulfate, $60 \mathrm{mM}(10.8 \mathrm{~g} / \mathrm{L})$ myo-inositol, and a certain amount of enzyme. The reaction system without $m y o$-inositol was pre-heated at $30^{\circ} \mathrm{C}$ for $10 \mathrm{~min}$ and then proceeded at $30{ }^{\circ} \mathrm{C}, 200 \mathrm{rpm}$ for $1 \mathrm{~h}$ after addition of myo-inositol. Then the reaction was stopped by boiling for $15 \mathrm{~min}$, and the supernatant was extracted after centrifugation at $12,000 \times g$ for $10 \mathrm{~min}$. The glucuronic acid concentration in the supernatant was determined by a chromogenic method using orcinol reagent $(100 \mathrm{~mL}$ of $37 \% \mathrm{HCl}, 0.1 \mathrm{~g}$ of orcinol, and $0.1 \mathrm{~g}$ of ferric trichloride). The supernatant and two times the volume of orcinol reagent were boiled together for $30 \mathrm{~min}$ and cooled to room temperature, after which $\mathrm{OD}_{660}$ was measured to quantify the glucuronic acid concentration [27]. The reaction system without myo-inositol was set as a control to determine MIOX4 activity. The protein concentration was determined by the Bradford method using BSA as a standard. One unit of MIOX4 was defined as the enzyme content needed to convert $1 \mu \mathrm{mol}$ myo-inositol in $1 \mathrm{~min}$ [28-30].

To analyze the expression level of MIOX4 protein, the Bga-2 recombinant strain was cultured in YPD medium to $\mathrm{OD}_{600}=0.8$, then inoculated into $50 \mathrm{~mL}$ of YPD medium and cultured for $48 \mathrm{~h}$. The whole cell protein was extracted to perform a Western blot, after which $15 \mu \mathrm{g}$ of total protein from each lysate was separated by $10 \%$ SDSPAGE and transformed onto PVDF membranes (Sangon Biotech, Shanghai, China). After blotting, the membranes were incubated overnight in a 1:2000 dilution of anti-HIS antibody according to the manufacturer's instructions. Western blot analysis was carried out as described previously [31].

\section{Relative quantification of gene expression levels}

To quantify gene expression levels, samples of approximately $10^{8}$ cells were harvested after inoculation for $16 \mathrm{~h}$. Total RNA was extracted from each of the samples using the RNAiso Plus RNA isolation kit (TaKaRa, Dalian, China) after liquid nitrogen grinding according to the manufacturer's instructions. Following genomic DNA eraser treatment to remove DNA, cDNA was synthesized from $500 \mathrm{ng}$ of total RNA using random primers with the PrimerScript RT reagent kit. The synthesized cDNA was then amplified in a quantitative PCR (qPCR) with primers Miox-a/Miox-s, Udh-a/Udh-s, and Pgk-a/Pgk-s respectively for relative quantification of mRNA levels of miox4 and $u d h$, using the phosphoglucokinase gene (PGK1) as an internal control. qPCR reactions containing SYBR Premix Ex Taq were performed in a Thermo Scientific CFX96 instrument. Each reaction was carried out in triplicate, and the reported $\mathrm{Ct}$ value was the average of the triplicate samples. Transcript levels were calculated using the $-\Delta \Delta \mathrm{Ct}$ method [32].

\section{Fed-batch fermentation and metabolite analysis}

Slant culture was inoculated into $60 \mathrm{~mL}$ YPD medium and cultured at $30^{\circ} \mathrm{C}, 250 \mathrm{rpm}$ for $24 \mathrm{~h}$ to the exponential growth phase. Before fermentation, the rotation speed, ventilation volume, and DO were set to $600 \mathrm{rpm}, 3 \mathrm{~L} /$ min, and $100 \%$ respectively. Then $60 \mathrm{~mL}$ seed culture was inoculated into a 5-L bioreactor containing $3 \mathrm{~L}$ fermentation medium, where $\mathrm{pH}$ was controlled at $4 \pm 0.1$ using $2.0 \mathrm{M} \mathrm{HCl}$ and $2.0 \mathrm{M} \mathrm{NaOH}$ and $\mathrm{DO}$ was controlled at $50 \%$ at $30{ }^{\circ} \mathrm{C}$.

Fermentation samples were taken, filtered through a $0.22-\mu \mathrm{m}$ membrane, and subjected to high-performance liquid chromatography (HPLC). Glucaric acid, lactic acid, ethanol, acetic acid, and glucose concentration were quantified by a Hitachi Chromaster using an Aminex HPX-87H column $(300 \mathrm{~mm} \times 7.8 \mathrm{~mm}$; Bio-Rad Laboratories, Hercules, CA). Sulfuric acid ( $5 \mathrm{mM})$ was used as the mobile phase at $30{ }^{\circ} \mathrm{C}$ and a flow rate of $0.6 \mathrm{~mL} / \mathrm{min}$ in isocratic mode. Compounds were detected from $30-\mu \mathrm{L}$ injections using a refractive index detector.

\section{Results \\ Construction of the biosynthetic pathway to produce glucaric acid in Saccharomyces cerevisiae}

To construct the glucaric acid biosynthetic pathway, a pY26-miox4-udh plasmid expressing miox4 and $u d h$ genes under control of strong promoters GPD and TEF [33] respectively was constructed and transformed into $S$. cerevisiae strain BY4741 opi1s, generating strain Bga-1. The Bga-1 strain was cultivated in YPD medium supplemented with $60 \mathrm{mM}(10.8 \mathrm{~g} / \mathrm{L})$ myo-inositol [34], where glucaric acid should be detected if miox4 and $u d h$ were both successfully expressed. As expected, glucaric acid was successfully detected in the culture supernatant of the Bga-1 strain by LC-MS. The highest production of glucaric acid was $0.54 \mathrm{~g} / \mathrm{L}$ in YPD medium containing $60 \mathrm{mM}(10.8 \mathrm{~g} / \mathrm{L})$ myo-inositol after 240 -h shake flask cultivation (Fig. 2a). However, no glucaric acid was produced when no myo-inositol was supplemented into the culture medium, which indicated that the myo-inositol 

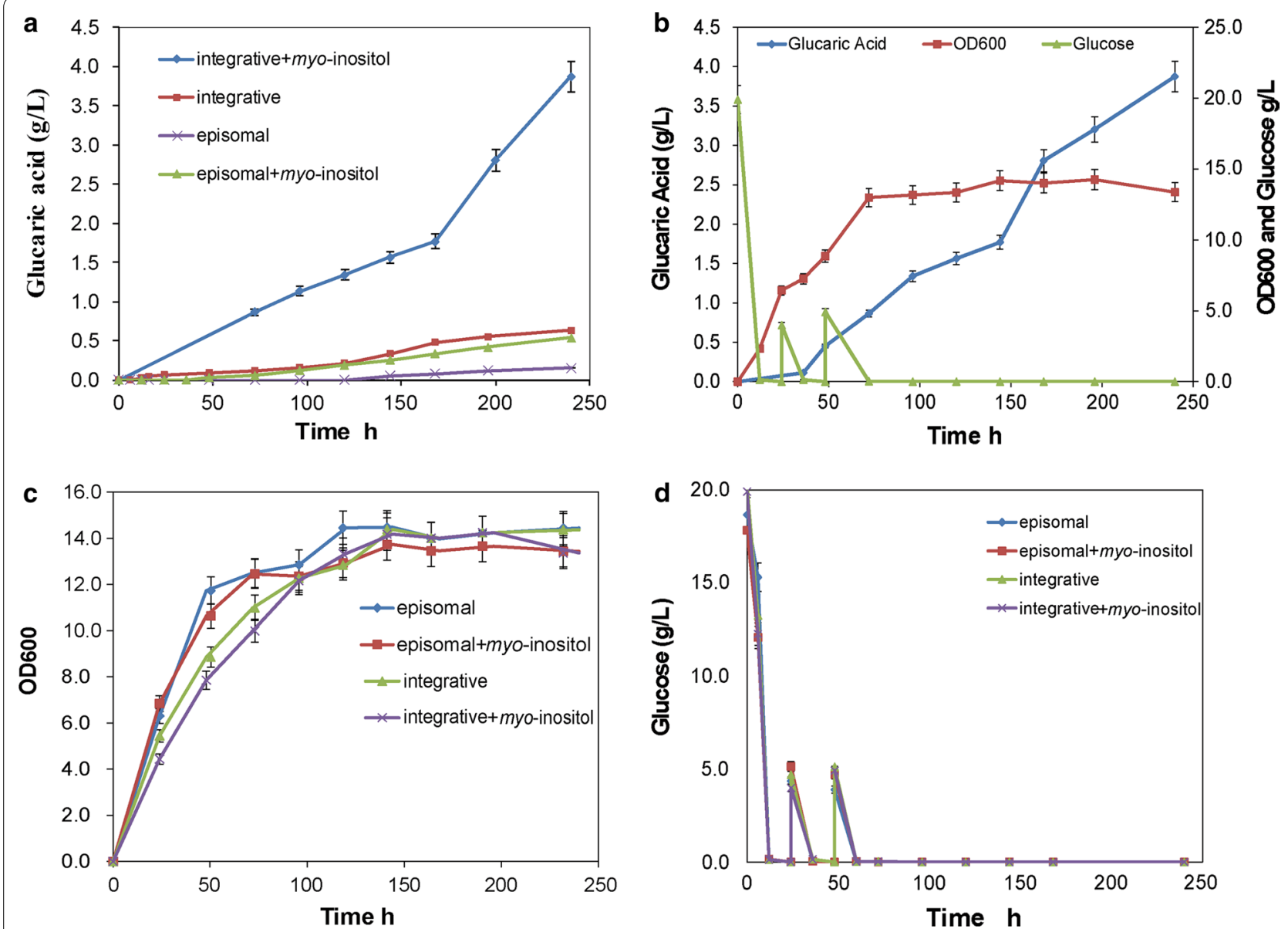

Fig. 2 Fermentation results for glucaric acid by episomal and integrative expression strains. a Glucaric acid titer of episomal and integrative expression strains in YPD medium with and without myo-inositol. b Glucaric acid production of the integrative strain (Bga-3) in YPD medium with myo-inositol. c Growth of episomal and integrative expression strains in YPD medium with and without myo-inositol. d Glucose consumption of episomal and integrative expression strains in YPD medium with and without myo-inositol. All experiments were performed in triplicate, and the error bars represent mean \pm standard deviation

produced by the yeast cell itself was not enough to produce glucaric acid.

\section{miox4 derived from Arabidopsis thaliana}

MIOX was first reported in 1957, when Charalampous et al. [27] found that one kind of enzyme that can convert myo-inositol to glucuronic acid existed in the mouse kidney. Reddy et al. [28, 29] isolated MIOX from hog kidney and found that MIOX could be activated after incubation in $\mathrm{Fe}^{2+}$ and cysteine, which solved the instability problem for this enzyme [9]. In addition, Moon et al. [1] synthesized the gene sequence of mouse-derived MIOX, cloned it into pTrc plasmid, and transformed it into $E$. coli BL21 (DE3) for expression. Although enzyme activity reached $0.43 \mathrm{U} / \mathrm{mg}$, MIOX was found to be extremely unstable. Activity reached a maximum after culturing in a medium containing $60 \mathrm{mM}(10.8 \mathrm{~g} / \mathrm{L})$ of myo-inositol, but then sharply declined, making MIOX a rate-limiting step in the glucaric acid synthetic pathway. Gupta et al. constructed a glucaric acid synthetic pathway in S. cerevisiae using MIOX from both M. musculus (Mm strain) and $A$. thaliana (At strain). They found that the At strain produced more glucaric acid $(0.56 \mathrm{~g} / \mathrm{L})$ than the $\mathrm{Mm}$ strain $(0.29 \mathrm{~g} / \mathrm{L})$, but glucaric acid production was still limited by both MIOX activity and myo-inositol availability during various growth stages. The authors of this paper believe that MIOX from A. thaliana is more stable than that from $M$. musculus [8]. Therefore, the more stable myo-inositol oxygenase MIOX4 [10] derived from A. thaliana was chosen in this study. To confirm MIOX4 activity, the pY26-miox4-histag plasmid was constructed, which could express the MIOX4- $6 \times$ His fusion protein and transform it to BY4741 opi1A, generating the Bga-2 strain. Western blot analysis revealed that the MIOX4 protein was successfully expressed in S. cerevisiae with a molecular weight of $37 \mathrm{kDa}$ [34] (Fig. 3a). MIOX4 protein 

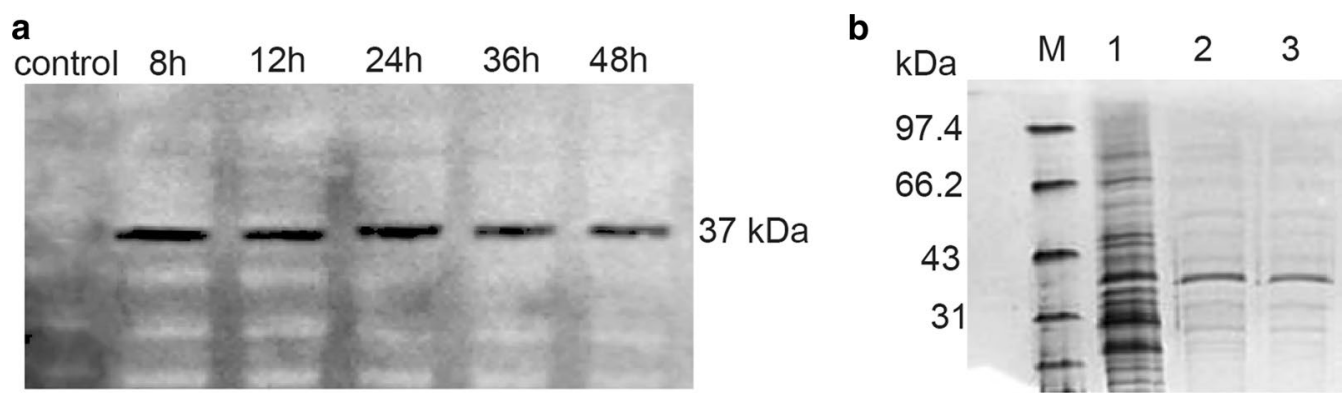

Fig. 3 MIOX4 protein analysis. a Western blot analysis of MIOX4. Whole-cell protein was extracted to perform Western blot after the recombinant strain Bga-2 was cultured in YPD medium with $60 \mathrm{mM}(10.8 \mathrm{~g} / \mathrm{L})$ myo-inositol for the indicated time. b SDS-PAGE analysis of MIOX4. To purify the MIOX4 protein, the His-tag was fused to the C-terminal of MIOX4. Strains were cultured as above, and the whole-cell protein was extracted and purified with the $6 \times$ His-Tagged Protein Purification Kit. The purified protein was verified by SDS-PAGE. Lane 1: protein marker, Lane 2: control (BY4741 carrying pY26 plasmid), Lanes 3 and 4: Bga-2

purification [35] by a Ni-column affinity chromatography kit also indicated that MIOX4 was successfully expressed in S. cerevisiae (Fig. 3b). After deletion of the OPI1 gene, S. cerevisiae accumulated $0.4 \mathrm{~g} / \mathrm{L}$ of myo-inositol, and the MIOX activity was much higher in the opi1 mutant than that of the wild-type cells (Additional file 1: Figure S1), which indicated that the deletion of OPI1 gene played a significant role in maintaining MIOX4 activity [20,21].

The BY4741 S. cerevisiae strain containing pY26miox4-histag was cultured in YPD medium for 7 days. The protein was extracted, adjusted to the desired concentration, and subjected to a Western blot test. MIOX4 protein expression decreased slightly in $36 \mathrm{~h}$ (Fig. 3a), whereas the enzymatic activity of MIOX4 declined after $48 \mathrm{~h}$ of MIOX4 protein expression, but became stable in the following 6 days (Fig. 4). These results showed that $A$. thaliana-derived MIOX4 was more efficient and stable in S. cerevisiae than M. musculus-derived MIOX expressed in E. coli [1].

\section{Increased glucaric acid titer through delta-sequence-based integrative expression}

It was suspected that the main reason for low glucaric acid titer was the lower expression level of miox4 and $u d h$ genes in plasmid episomal expression, especially the miox4 gene. To circumvent plasmid instability

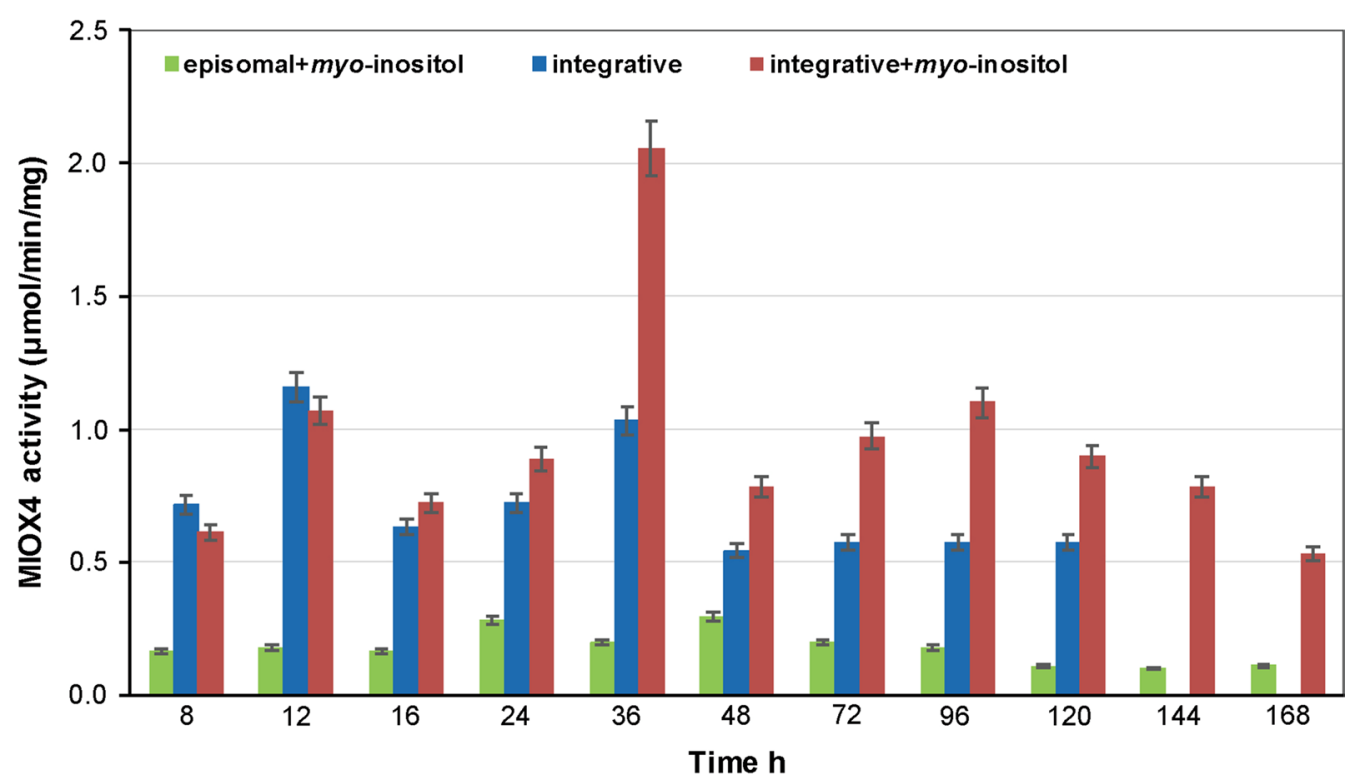

Fig. 4 MIOX4 activity in the episomal expression strain with myo-inositol and the integrative expression with and without myo-inositol. All experiments were performed in triplicate, and the error bars represent mean \pm standard deviation 
and increase the number of target gene copies, a delta sequence-based constitutive expression method was used to overexpress target genes [25]. S. cerevisiae delta sequences are long terminal repeats of transposons Ty1 and Ty2, and a total of 425 delta sequences are dispersed through the yeast genome [17]. miox4 and $u d h$ were integrated under control of GPD and TEF promoters [33] respectively into the chromosome of $S$. cerevisiae BY4741 opi1A through delta sequence-based homologous recombination (Fig. 1). Following successful integration, one strain with the highest titer was selected and named S. cerevisiae BY4741 opi1L-mu (Bga-3). This strain produced $3.8 \mathrm{~g} / \mathrm{L}(18.1 \mathrm{mM})$ glucaric acid in shake flask culture with $60 \mathrm{mM}(10.8 \mathrm{~g} / \mathrm{L})$ myo-inositol, which was 7.04 times higher than that of the Bga-2 episomal plasmid expression in S. cerevisiae (Fig. 2b). Interestingly, there were no obvious differences in growth rate or glucose consumption between the Bga- 2 episomal plasmid expression strain and the Bga-3 integrative expression strain with or without myo-inositol (Fig. 2c, d).
YPD medium supplemented with $0,10 \mathrm{mM}(1.8 \mathrm{~g} / \mathrm{L})$ or $60 \mathrm{mM}(10.8 \mathrm{~g} / \mathrm{L})$ of $m y o$-inositol was used to produce glucaric acid. The results showed the highest glucaric acid titer generated in $60 \mathrm{mM}(10.8 \mathrm{~g} / \mathrm{L})$ myo-inositol (Fig. 5a), suggesting that myo-inositol availability was rate-limiting in glucaric acid synthesis. Then $5 \mathrm{~g} / \mathrm{L}$ of glucose was fed once at $12,24,48 \mathrm{~h}$ respectively, or fed twice at $12 / 24$ and $24 / 48 \mathrm{~h}$. The results showed that glucose fed twice at 24/48 $\mathrm{h}$ was best for increasing glucaric acid production (Fig. 5b). Henry et al. [36] reported that the exponential growth phase was the best time for myo-inositol accumulation, which further increased glucaric acid titer. The glucose feeding concentration was also tested. 5,10 , and $20 \mathrm{~g} / \mathrm{L}$ of glucose was fed at 24 and $48 \mathrm{~h}$; the results showed that the highest glucaric acid titer occurred at $5 \mathrm{~g} / \mathrm{L}$ of glucose, but that higher glucose feeding contents had a negative effect on glucaric acid titer (Fig. 5c). The reason why more glucose decreased glucaric acid titer was suspected to be that excessive glucose fluxed into the glycolytic pathway to increase biomass, instead of
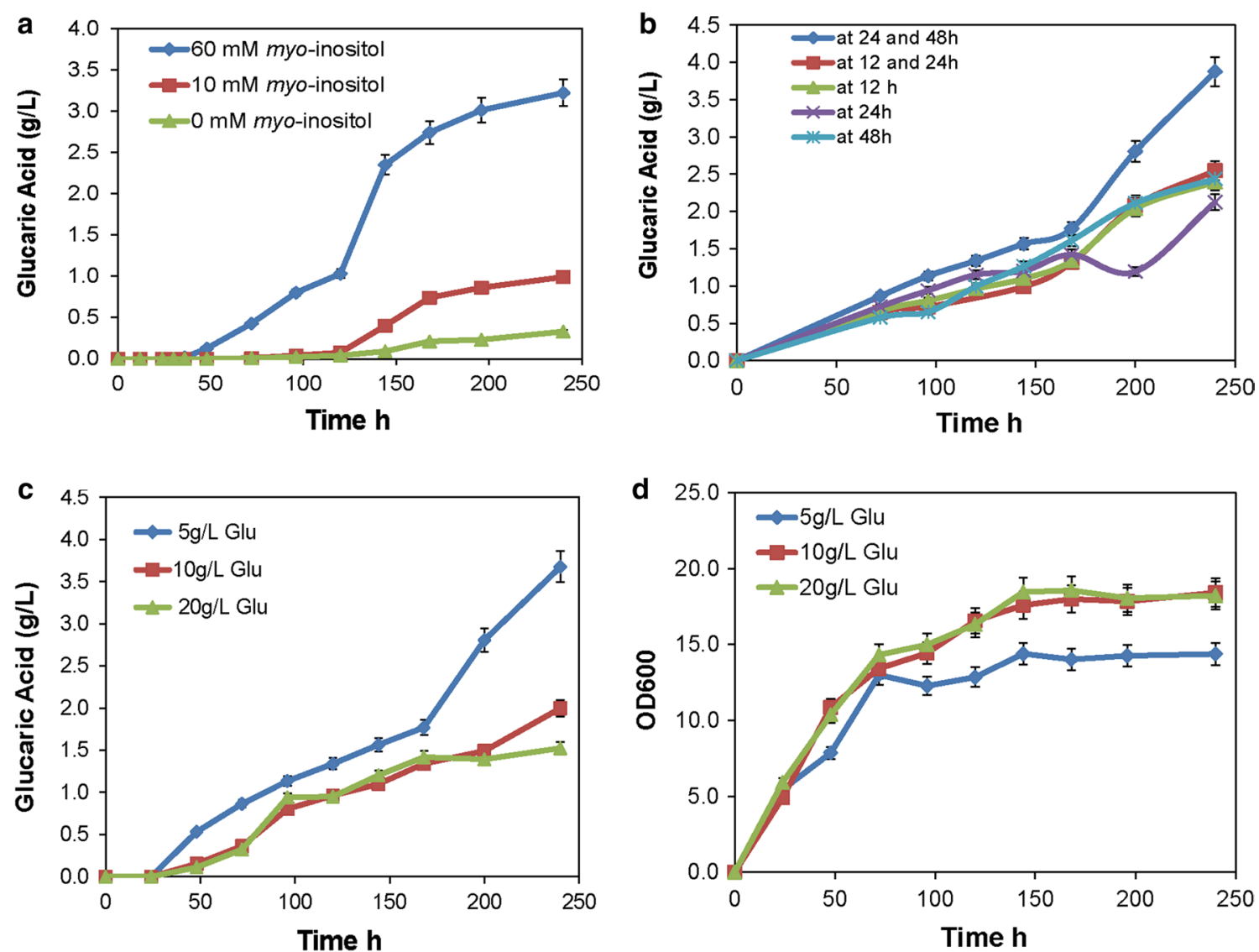

Fig. 5 Fermentation results for glucaric acid by strain Bga-3. a Glucaric acid produced in YPD medium with variable myo-inositol concentrations. b Glucaric acid produced in YPD medium supplemented with 60 mM (10.8 g/L) myo-inositol at different points in time. c Glucaric acid produced in YPD medium with variable glucose concentrations. $\mathbf{d}$ Growth profile of Bga-3 in YPD medium with variable glucose concentrations. All experiments were performed in triplicate, and the error bars represent mean \pm standard deviation 
myo-inositol (Fig. 5d). Therefore, the best fermentation condition was YPD medium supplemented with $60 \mathrm{mM}$ $(10.8 \mathrm{~g} / \mathrm{L})$ myo-inositol, and with $5 \mathrm{~g} / \mathrm{L}$ of glucose fed at 24 and $48 \mathrm{~h}$. Under this culture condition, the highest glucaric acid titer $[3.8 \mathrm{~g} / \mathrm{L}(18.1 \mathrm{mM})]$ was produced in the shake flask, with a $1.76 \pm 0.08 \mathrm{~g} / \mathrm{g}$-myo-inositol yield and a $0.127 \pm 0.005 \mathrm{~g} / \mathrm{g}$-glucose yield.

\section{Increased MIOX4 activity and gene expression level by multi-copy integrative expression}

To determine whether the high glucaric acid yield was due to the high expression levels of miox4 and $u d h$, quantitative real-time PCR (RT-qPCR) was performed to confirm whether delta sequence-based integrative expression increased gene expression levels of miox 4 and

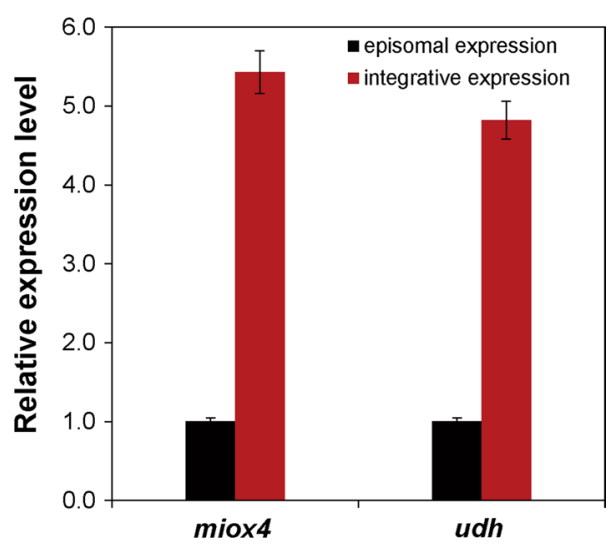

Fig. 6 Relative gene expression levels of miox 4 and $u d h$. All experiments were performed in triplicate, and the error bars represent mean \pm standard deviation udh compared with episomal plasmid expression. The result showed that the transcription levels of miox4 and $u d h$ from integrative expression were 5.43 and 4.82 times of those from episomal expression (Fig. 6). MIOX4 enzymatic activities of integrative and episomal expression with and without myo-inositol were also measured. The specific activity of MIOX4 in integrative expression with myo-inositol reached a maximum of $2.05 \mathrm{U} / \mathrm{mg}$, then declined, but remained above $0.5 \mathrm{U} / \mathrm{mg}$. In contrast, the highest specific activity of MIOX4 in episomal expression with $m y o$-inositol was $0.295 \mathrm{U} / \mathrm{mg}$ (Fig. 4), suggesting that multi-copy integrative MIOX4 increased enzymatic activity more than episomal expression by increasing mRNA transcription levels. In addition, MIOX4 activity in integrative expression with myo-inositol was more efficient and stable than without myo-inositol, which confirmed the extreme importance of myo-inositol to the activity of myo-inositol oxygenase. This study showed that regardless of episomal plasmid expression or integrative expression, the MIOX4 activity decreased steadily, but remained above a certain value. Unlike E. coli, $S$. cerevisiae has an endogenous myo-inositol accumulation pathway, thus increasing the glucaric acid production.

\section{Fed-batch fermentation}

To provide further confirmation of the performance of the multi-copy episomal expression strain as constructed, fed-batch fermentation in a 5-L bioreactor was carried out. As DO and $\mathrm{pH}$ were controlled at their optimal values for S. cerevisiae growth, the BY4741 opi1A-mu (Bga-3) strain produced glucaric acid up to $6.0 \mathrm{~g} / \mathrm{L}$ $(28.6 \mathrm{mM})$ at $216 \mathrm{~h}$ (Fig. $7 \mathrm{a}$ ) with only a few by-products of ethanol, acetic acid, and lactic acid (Fig. 7b). This was the highest titer ever reported in S. cerevisiae, with a
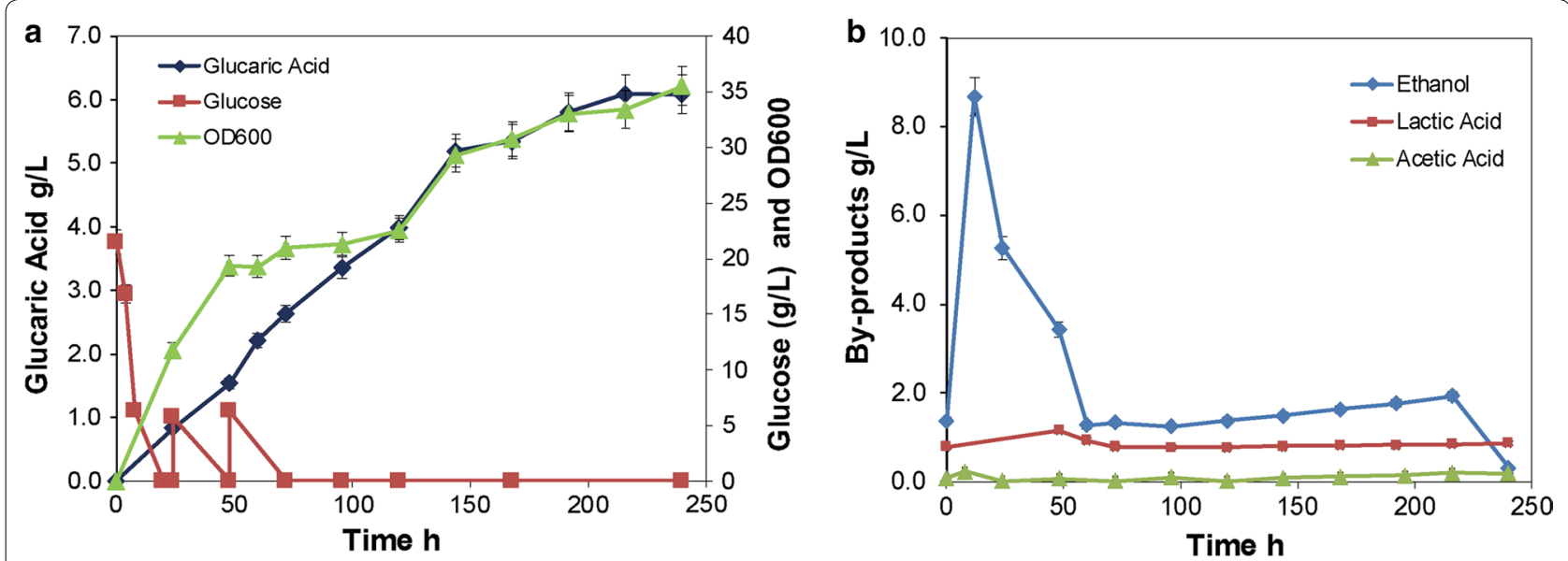

Fig. 7 Fed-batch fermentation of glucaric acid by the Bga-3 strain. a Growth, glucose consumption, and glucaric acid production. $\mathbf{b}$ Byproducts production. All experiments were performed in triplicate, and the error bars represent mean \pm standard deviation 
$1.67 \pm 0.08 \mathrm{~g} / \mathrm{g}$-myo-inositol yield and a $0.2 \pm 0.008 \mathrm{~g} / \mathrm{g}$ glucose yield. The results obtained showed that the byproduct ethanol and lactic acid accumulated in the initial stage, but declined in the later phase of fermentation. This indicated that the byproduct ethanol and lactic acid may be partially utilized by the yeast cells, as reported previously [37-39].

\section{Discussion}

Cell factories created by metabolic engineering and synthetic biology to produce fine chemicals have emerged as an increasingly popular alternative to chemical synthesis [40]. Ever since the glucaric acid biosynthetic pathway was constructed in E. coli [1], great efforts have been expended to increase target product titer. Gupta et al. [8] reported that MIOX activity and myo-inositol availability were rate-limiting in glucaric acid production, not only in E. coli, but in S. cerevisiae. Therefore, the strategy to increase glucaric acid production was to improve MIOX activity and stability while increasing the myo-inositol flux to glucaric acid.

In $S$. cerevisiae, myo-inositol can be produced by converting D-glucose-6-phosphate by native inositol1-phosphate synthase (Ino1), which is tightly regulated by Opi1 [36]. In this study, the OPI1 gene was deleted to remove its negative regulation of myo-inositol synthesis. Although more myo-inositol was produced by $S$. cerevisiae, exogenous supplementation of myo-inositol was still necessary to increase MIOX4 activity (Fig. 2a) and glucaric acid accumulation. In addition, the constructed strain supplemented with $60 \mathrm{mM}(10.8 \mathrm{~g} / \mathrm{L})$ myo-inositol apparently produced more glucaric acid than $10 \mathrm{mM}$ $(1.8 \mathrm{~g} / \mathrm{L})$ myo-inositol (Fig. 5a), which indicated that efficient production of glucaric acid at high titer needs a high concentration of myo-inositol might because the low affinity activity of the Itr $1 / 2$ myo-inositol transporter in S. cerevisiae [41]. However, only a small proportion of the supplemented myo-inositol was transported into the cell (Additional file 1: Figure S2). When the myo-inositol residue was fed $60 \mathrm{mM}(10.8 \mathrm{~g} / \mathrm{L})$ myo-inositol to a shake flask culture, only about $20 \%(2.16 \pm 0.11 \mathrm{~g} / \mathrm{L})$ of myoinositol was consumed (Additional file 1: Figure S2A). At least $3.26 \mathrm{~g} / \mathrm{L}$ myo-inositol was required to produce $3.8 \mathrm{~g} / \mathrm{L}$ glucaric acid in the shake flask culture. This means that at least $1.1 \mathrm{~g} / \mathrm{L}$ myo-inositol was endogenously synthesized from glucose; the yields were $1.76 \pm 0.08 \mathrm{~g}$ glucaric acid/g myo-inositol and $0.037 \pm 0.003 \mathrm{~g}$ myoinositol/g glucose. In the fed-batch fermentation of glucaric acid, about $3.6 \pm 0.18 \mathrm{~g}$ myo-inositol was consumed (Additional file 1: Figure S2B), and about $5.14 \mathrm{~g} / \mathrm{L}$ myoinositol was required to produce $6.0 \mathrm{~g} / \mathrm{L}$ glucaric acid. Therefore, at least $1.54 \mathrm{~g} / \mathrm{L}$ myo-inositol came from glucose, and the yield was $1.67 \pm 0.08 \mathrm{~g}$ glucaric acid $/ \mathrm{g}$ myo-inositol, or $0.051 \pm 0.006 \mathrm{~g}$ myo-inositol/g glucose. Robinson reported that the uptake activity of the Itr1p myo-inositol transporter was affected by growth phase in $S$. cerevisiae because it would reach a maximum at exponential and then decrease rapidly to minimum in the stationary phase, and this regulation was independent of OPI1 [41]. Improving the transport ability of Itr1p through protein engineering may be very important.

Besides myo-inositol availability, low activity or instability of myo-inositol oxygenase is the key bottleneck in the biosynthetic pathway to glucaric acid. Given that the codon-optimized mouse-derived MIOX gene had low activity and instability according to Moon et al. [1], the miox4 gene from A. thaliana was selected for this study. MIOX4 showed relatively high stability compared with mouse MIOX, but the specific activity of episomal plasmid expression of miox 4 was still very slow. To overcome this problem, delta-sequence-based integrative expression of miox4 and $u d h$ genes was carried out. A delta sequence is a kind of long-end repeated DNA sequence located in the reverse transcription transposon Ty of chromosome DNA in S. cerevisiae. Because there are approximately 425 delta sequences, delta sequence-based homologous recombination is more efficient than traditional methods for exogenous DNA integration into the $S$. cerevisiae genome, and the target gene was expressed more stably in the delta sequence because it avoided plasmid loss in the episomal expression strain [17]. The miox4 transcription level as determined by quantitative real-time PCR was an indirect characterization of gene copies compared with that of the episomal plasmid. The transcription level of miox4 in integrative expression was 5.43 times that of episomal expression (Fig. 6). These results indicated that this multi-copy integrative expression method is efficient for overexpression of miox4 genes, resulting in obviously enhanced MIOX4 activity and stability and high glucaric acid titer. The authors believe that the delta-sequence integration method can be used in a wide range of low-copy genes to remove the bottleneck in metabolic engineering of S. cerevisiae.

\section{Conclusions}

In this study, glucaric acid titer in S. cerevisiae was increased by expressing a more stable MIOX4 from $A$. thaliana and integrating the target genes into the delta sequence of the genomes. Delta-sequence-based constitutive expression increased both the number of target gene copies and their stability and can be used for a wide range of metabolic pathway engineering projects in $S$. cerevisiae. The final strain produced $6.0 \mathrm{~g} / \mathrm{L}(28.6 \mathrm{mM})$ glucaric acid, which is the highest titer reported in $S$. cerevisiae. 


\section{Additional file}

Additional file 1: Table S1. Primers used in this study. Figure S1. MIOX4 activity of the episomal expression plasmid in the wild-type strain and opil mutant strain with myo-inositol. All experiments were performed in triplicates and the error bar represented mean \pm standard deviation. Figure S2. myo-inositol residue in shake flask (A) and fed-batch (B) cultures when fed $60 \mathrm{mM}(10.8 \mathrm{~g} / \mathrm{L})$ myo-inositol to the culture. All experiments were performed in triplicates and the error bar represented mean \pm standard deviation.

\section{Authors' contributions}

YD and YZ contributed to designing the experiments, analyzing the data, and writing the manuscript. NC and JW performed construction of plasmids and yeast strains and fermentation and enzyme assays. All authors read and approved the final manuscript.

\section{Author details}

${ }^{1}$ National Engineering Laboratory for Cereal Fermentation Technology (NELCF), Jiangnan University, 1800 Lihu Road, Wuxi 214122, Jiangsu, China. ${ }^{2}$ School of Biotechnology, Jiangnan University, 1800 Lihu Road, Wuxi 214122, Jiangsu, China.

\section{Acknowledgements}

We thank the Key Laboratory of Industrial Biotechnology, Ministry of Education, Jiangnan University for LC-MS analysis. We thank International Science Editing (http://www.internationalscienceediting.com) for editing this manuscript.

\section{Competing interests}

The authors declare that they have no competing interests.

\section{Consent for publication}

The manuscript is original, has not already been published, and is not currently under consideration by another journal.

\section{Ethics approval and consent to participate} Not applicable.

\section{Funding}

This work was supported by Grants from the National Natural Science Foundation of China (31500070), the Natural Science Foundation of Jiangsu Province (BK20150136, BK20150151), and the Fundamental Research Funds for the Central Universities (JUSRP51705A).

\section{Publisher's Note}

Springer Nature remains neutral with regard to jurisdictional claims in published maps and institutional affiliations.

Received: 3 January 2018 Accepted: 24 April 2018

Published online: 05 May 2018

\section{References}

1. Moon TS, Yoon SH, Lanza AM, Roy-Mayhew JD, Prather KL. Production of glucaric acid from a synthetic pathway in recombinant Escherichia coli. Appl Environ Microbiol. 2009;75:589-95.

2. Walaszek Z, Szemraj J, Hanausek M, Adams AK, Sherman U. D-Glucaric acid content of various fruits and vegetables and cholesterol-lowering effects of dietary D-glucarate in the rat. Nutr Res. 1996;16:673-81.

3. Singh J, Gupta KP. Induction of apoptosis by calcium D-glucarate in 7,12-dimethyl benz [a] anthracene-exposed mouse skin. J Environ Pathol Toxicol Oncol. 2007;26:63-73.

4. Donald EK, Chen L, Lin TH. Hydroxylated nylons based on unprotected esterified D-glucaric acid by simple condensation reactions. J Am Chem Soc. 1993;116:571-8.
5. Smith TN, Hash K, Davey CL, Mills H, Williams H, Kiely DE. Modifications in the nitric acid oxidation of D-glucose. Carbohydr Res. 2012;350:6-13.

6. Moon TS, Dueber JE, Shiue E, Prather KL. Use of modular, synthetic scaffolds for improved production of glucaric acid in engineered $E$. coli. Metab Eng. 2010:12:298-305.

7. Shiue E, Prather KL. Improving D-glucaric acid production from myoinositol in E. coli by increasing MIOX stability and myo-inositol transport. Metab Eng. 2014;22:22-31.

8. Gupta A, Hicks MA, Manchester SP, Prather KL. Porting the synthetic D-glucaric acid pathway from Escherichia coli to Saccharomyces cerevisiae. Biotechnol J. 2016;11:1201-8.

9. Liu Y, Gong X, Wang C, Du G, Chen J, Kang Z. Production of glucaric acid from myo-inositol in engineered Pichia pastoris. Enzyme Microb Technol. 2016;91:8-16.

10. Lorence A, Chevone BI, Mendes P, Nessler CL. myo-inositol oxygenase offers a possible entry point into plant ascorbate biosynthesis. Plant Physiol. 2004;134:1200-5.

11. Smirnoff N, Wheeler GL. Ascorbic Acid in plants: biosynthesis and function. Crit Rev Plant Sci. 2000;19:267-90.

12. Wong L, Engel J, Jin E, Holdridge B, Xu P. YaliBricks, a versatile genetic toolkit for streamlined and rapid pathway engineering in Yarrowia lipolytica. Metab Eng Commun. 2017;5:68-77.

13. Oba T, Suenaga H, Nakayama S, Mitsuiki S, Kitagaki H, Tashiro K, Kuhara S. Properties of a high malic acid-producing strains of Saccharomyces cerevisiae isolated from sake mash. Biosci Biotechnol Biochem. 2011;75:2025-9.

14. Yan D, Wang C, Zhou J, Liu Y, Yang M, Xing J. Construction of reductive pathway in Saccharomyces cerevisiae for effective succinic acid fermentation at low pH value. Bioresour Technol. 2014;156:232-9.

15. Xu P, Qiao KJ, Ahn WS, Stephanopoulos G. Engineering Yarrowia lipolytica as a platform for synthesis of drop-in transportation fuels and oleochemicals. Proc Natl Acad Sci USA. 2016:113:10848-53.

16. Xu P, Qiao K, Stephanopoulos G. Engineering oxidative stress defense pathways to build a robust lipid production platform in Yarrowia lipolytica. Biotechnol Bioeng. 2017;114:1521-30.

17. Lee FWF, Silva NAD. Improved efficiency and stability of multiple cloned gene insertions at the $d$ sequences of Saccharomyces cerevisiae. Appl Microbiol Biotechnol. 1997:48:339-45.

18. Yoon SH, Moon TS, Iranpour P, Lanza AM, Prather KJ. Cloning and characterization of uronate dehydrogenases from two pseudomonads and Agrobacterium tumefaciens strain C58. J Bacteriol. 2009;191:1565-73.

19. Chen FJ, Zhou JW, Shi ZP, Liu LM, Du GC, Chen J. Effect of acetyl-CoA synthase gene overexpression on physiological function of Saccharomyces cerevisiae. Acta Microbiol Sin. 2010;50:1172-9.

20. Graves JA, Henry SA. Regulation of the yeast INO1 gene: the products of INO2, INO4 and OPI1 regulatory genes are not required for repression in response to inositol. Genetics. 1999;154:1485-95.

21. Ye C, Bandara WM, Greenberg ML. Regulation of inositol metabolism is fine-tuned by inositol pyrophosphates in Saccharomyces cerevisiae. J Biol Chem. 2013;288:24898-908.

22. Hegemann JH, Heick SB. Delete and repeat: a comprehensive toolkit for sequential gene knockout in the budding yeast Saccharomyces cerevisiae. Methods Mol Biol. 2011;765:189-206.

23. Gietz RD, Schiestl RH. Frozen competent yeast cells that can be transformed with high efficiency using the LiAc/SS carrier DNA/PEG method. Nat Protoc. 2007:2:1-4.

24. Boeke JD, LaCroute F, Fink GR. A positive selection for mutants lacking orotidine-5'-phosphate decarboxylase activity in yeast: 5 -fluoro-orotic acid resistance. Mol Gen Genet. 1984;197:345-6.

25. Parekh RN, Shaw MR, Wittrup KD. An integrating vector for tunable, high copy, stable integration into the dispersed Ty delta sites of Saccharomyces cerevisiae. Biotechnol Prog. 1996;12:16-21.

26. Curran KA, Leavitt JM, Karim AS, Alper HS. Metabolic engineering of muconic acid production in Saccharomyces cerevisiae. Metab Eng. 2013;15:55-66.

27. Charalampous FC, Lyras C. Biochemical studies on inositol: IV. Conversion of inositol to glucuronic acid by rat kidney extracts. J Biol Chem. 1957;228:1-13.

28. Reddy CC, Pierzchala PA, Hamilton GA. myo-Inositol oxygenase from hog kidney: II. Catalytic properties of the homogeneous enzyme. J Biol Chem. 1981;256:8519-24. 
29. Reddy CC, Swan JS, Hamilton GA. myo-Inositol oxygenase from hog kidney: I. Purification and characterization of the oxygenase and of an enzyme containing the oxygenase and D-glucuronate reductase. J Biol Chem. 1981;256:8510-8.

30. Arner RJ, Prabhu KS, Thompson JT, Hildenbrandt GR, Liken AD, Reddy CC. myo-Inositol oxygenase: molecular cloning and expression of a unique enzyme that oxidizes myo-inositol and D-chiro-inositol. Biochem J. 2001;360:313-20.

31. Miller-Fleming $L_{1}$ Cheong $H$, Antas $P$, Klionsky DJ. Detection of Saccharomyces cerevisiae Atg13 by western blot. Autophagy. 2014;10:514-7.

32. Livak KJ, Schmittgen TD. Analysis of relative gene expression data using real-time quantitative PCR and the 2(-Delta Delta C(T)) Method. Methods. 2001;25:402-8.

33. Mumberg D, Muller R, Funk M. Yeast vectors for the controlled expression of heterologous proteins in different genetic backgrounds. Gene. 1995;156:119-22.

34. Arner RJ, Prabhu KS, Reddy CC. Molecular cloning, expression, and characterization of myo-inositol oxygenase from mouse, rat, and human kidney. Biochem Biophys Res Commun. 2004;324:1386-92.

35. Cuatrecasas P. Protein Purification by affinity chromatography: derivatizations of agarose and polyacrylamide beads. J Biol Chem. 1970;245:3059-65.
36. Henry SA, Gaspar ML, Jesch SA. The response to inositol: regulation of glycerolipid metabolism and stress response signaling in yeast. Chem Phys Lipids. 2014;180:23-43.

37. van de Laar T, Visser C, Holster M, Lopez CG, Kreuning D, Sierkstra L, Lindner N, Verrips T. Increased heterologous protein production by Saccharomyces cerevisiae growing on ethanol as sole carbon source. Biotechnol Bioeng. 2007;96:483-94.

38. Flores CL, Rodriguez C, Petit T, Gancedo C. Carbohydrate and energyyielding metabolism in non-conventional yeasts. FEMS Microbiol Rev. 2000;24:507-29.

39. Casal M, Cardoso H, Leao C. Mechanisms regulating the transport of acetic acid in Saccharomyces cerevisiae. Microbiology. 1996;142:1385-90.

40. Xu P. Production of chemicals using dynamic control of metabolic fluxes. Curr Opin Biotechnol. 2017;53:12-9.

41. Robinson KS, Lai K, Cannon TA, McGraw P. Inositol transport in Saccharomyces cerevisiae is regulated by transcriptional and degradative endocytic mechanisms during the growth cycle that are distinct from inositolinduced regulation. Mol Biol Cell. 1995;7:81-9.
Ready to submit your research? Choose BMC and benefit from:

- fast, convenient online submission

- thorough peer review by experienced researchers in your field

- rapid publication on acceptance

- support for research data, including large and complex data types

- gold Open Access which fosters wider collaboration and increased citations

- maximum visibility for your research: over 100M website views per year

At BMC, research is always in progress.

Learn more biomedcentral.com/submissions 